\title{
Pole and shape of (1459) Magnya, the outer main belt basaltic asteroid ${ }^{\star}$ (Research Note)
}

\author{
J. S. Silva and D. Lazzaro
}

\author{
Observatório Nacional, rua Gal. José Cristino 77, 20921-400 Rio de Janeiro, Brazil \\ e-mail: jsergio@on.br
}

Received 18 April 2015 / Accepted 22 June 2015

\begin{abstract}
Context. The basaltic asteroid (1459) Magnya is, to date, the only confirmed example of this kind of asteroid in the outer main belt (Lazzaro et al. 2000). It might be a rare surviving fragment from a larger, differentiated, and subsequently disrupted object most probably unrelated to (4) Vesta.

Aims. We derive the direction of the pole and the shape of asteroid (1459) Magnya in order to increase the knowledge of the body's physical properties. In particular, these two properties are useful to characterize which regions of the body are being sampled by spectral observations at distinct epochs and telescopes and to better assess the degree of surface homogeneity of (1459) Magnya. Methods. An observational campaign of (1459) Magnya was set up to determine its pole and shape from photometric lightcurves of the asteroid. These lightcurves were acquired at diverse telescopes and observatories from 2002 to 2014 . Additional data from the literature was added, and a method of inversion was applied.

Results. The obtained pole of asteroid (1459) Magnya is given by the ecliptic coordinates $\lambda=159 \pm 10^{\circ}$ and $\beta=-75 \pm 10^{\circ}$. The asteroid is modeled by a slightly elongated ellipsoid, rotating along the smallest axis with a period of $4.679100 \pm 0.000005 \mathrm{~h}$. The derived direction of the pole was used to compute the viewing geometry of the spectroscopic observations of (1459) Magnya obtained by various authors and indicates the necessity of new visible and near-infrared observations in order to correctly assess the degree of homogeneity of its surface.
\end{abstract}

Key words. minor planets, asteroids: individual: (1459) Magnya - techniques: spectroscopic - techniques: photometric methods: observational

\section{Introduction}

The discovery of a small basaltic asteroid in the outer main belt, (1459) Magnya, raised not only the problem of its origin (Lazzaro et al. 2000), but also that of the presence and extension of differentiated material in the asteroid belt. It is reckoned that the presence of a basaltic surface implies an extensive geochemical differentiation and resurfacing and according to our current understanding, this should occur only on large objects as a result of the heat needed to melt the chondritic material (Ruzicka et al. 1997). The only large asteroid in the main belt showing an almost intact basaltic crust is (4) Vesta, a $400 \mathrm{~km}$ diameter object located in the inner part of the belt, at $2.4 \mathrm{AU}$. Its basaltic nature was first inferred by McCord et al. (1970), and has been confirmed in all subsequent works and more recently in great detail by the Dawn mission (Russell et al. 2012).

The asteroid (1459) Magnya has an estimated diameter of just $17 \mathrm{~km}$ (Delbo et al. 2006), which implies that either it is a fragment of (4) Vesta or it is a remnant of the catastrophic disruption of another large basaltic object. For the first possibility to be true, the ejection velocity from (4) Vesta must have been larger than $5 \mathrm{~km} \mathrm{~s}^{-1}$ (Binzel \& Xu 1993; Lazzaro et al. 2000), which is quite unrealistic. The problem with the second

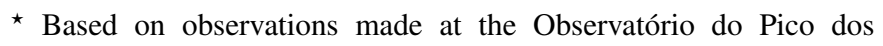
Dias/LNA, and at the Observatório Astronômico do Sertão de Itaparica/ON. possibility is the lack of an associated family. This problem can be overcome, however, if we assume that a family was formed and then dispersed. Indeed, the region around (1459) Magnya has been shown to be filled with high-order resonances that can disperse a family in a short timescale due to the slow chaotic diffusion of its members (Lazzaro et al. 2000; Michtchenko et al. 2002).

The basaltic composition of (4) Vesta, and of (1459) Magnya has been derived from spectroscopic observations that show the presence of two deep absorption bands, at 0.92-0.94 $\mu \mathrm{m}$ and at $2 \mu \mathrm{m}$, representative of this material. This composition is also similar to that of basaltic achondrite meteorites, specifically the Howardites, Eucrites, and Diogenites, collectively known as the HED suite of meteorites. It is noteworthy that given this similarity and the location of (4) Vesta near a transport route (3:1 mean motion and $v_{6}$ secular resonances with Jupiter), it is currently assumed that these meteorites do originate from (4) Vesta (Drake 2001).

Detailed near-infrared spectral observations of (1459) Magnya indicate the presence of pyroxenes which have a lower Fs level than those of (4) Vesta (Hardersen et al. 2004). This result strongly favors the hypothesis that (1459) Magnya originated from a parent body other than (4) Vesta. This hypothesis has been somewhat strengthened over the years by the discovery of small basaltic, or presumed basaltic, objects extending from the near-Earth region (Cruikshank et al. 1991) to the inner main 
belt, but outside the limits of Vesta's dynamical family (Xu et al. 1995; Burbine et al. 2001; Florczak et al. 2002; Lazzaro et al. 2004; Alvarez-Candal et al. 2006; Moskovitz et al. 2010), and in the intermediate belt (Binzel et al. 2006; Hammergren et al. 2007; Roig \& Gil-Hutton 2006) and outer belt (Lazzaro et al. 2000; Moskovitz et al. 2008).

Another important result from Hardersen et al. (2004) was that observations over $4.5 \mathrm{~h}$ showed little variation in band center positions indicating an almost homogeneous surface for (1459) Magnya. The determination of its rotational period $(4.68 \mathrm{~h}$; Almeida et al. 2004), confirmed that the observations indeed spanned an almost complete rotation of the asteroid. However, because the aspect angle (observer - spin axis) of the observations is not known, it is impossible to quantify the exact fraction of the sampled surface.

In order to better assess the degree of surface homogeneity of (1459) Magnya we decided to set up an observational campaign to derive the direction of its spin axis and an indication of its shape. It is noteworthy that these parameters are also important in order to schedule further observations of this very interesting asteroid. Photometric observations were performed in 2002, 2004, 2008, and 2014, spanning a $12 \mathrm{yr}$ time interval, and to these were added lightcurves from the literature. In what follows is given the description of the observations and the reduction process. The photometric analysis deriving the pole and shape of (1459) Magnya is then given in Sect. 3, while the last section is dedicated to a summary and a discussion of our findings.

\section{Observations and data reduction}

Photometric data of asteroid (1459) Magnya was acquired by our team at diverse telescopes and observatories during nine observing runs from 2002 to 2014. The April 2002 observations using the $1.6 \mathrm{~m}$ telescope at the Observatório do Pico dos Dias (OPD, Itajubá, Brazil) allowed the first determination of the asteroid rotational period $(4.68 \pm 0.01 \mathrm{~h}$; Almeida et al. 2004). Three other observing runs at the same observatory, but using the $0.6 \mathrm{~m}$ telescope, were performed in August and September, 2004, and in May 2008. In May, June, July, and August 2014, new observations were performed during five observing runs using the $1 \mathrm{~m}$ telescope of the IMPACTON project at the Observatorio Astronômico do Sertão de Itaparica (OASI, Itacuruba, Brazil).

All observations used CCD cameras, in the $V$ band at OPD and $R$ band at OASI. In the observations at the OPD with the $0.6 \mathrm{~m}$ telescope a EEV $385 \times 576 \mathrm{CCD}$ was used, with focalplane reducing optics, giving a $7.2 \times 5.50$ arcmin field and yielding an image scale of $1.12 \mathrm{arcsec} / \mathrm{pix}$. The $1.6 \mathrm{~m}$ telescope was used with a $1024 \times 1024 \mathrm{CCD}$ with an image scale of 0.31 arcsec/pix, giving a $5.3 \times 5.3$ arcmin field. The observations at OASI were made using a $1024 \times 1024$ Apogee Alta U47 CCD camera, giving a $5.9 \times 5.9$ arcmin field and an image scale of $0.343 \mathrm{arcsec} / \mathrm{pix}$.

The data reduction was done using the MaxIm DL package and science targets were reduced following the standard procedures of flat-field correction and sky subtraction and all the times were corrected for light-travel time. Relative magnitudes, between the asteroid and a field star of similar magnitude, were computed yelding 16 individual and 9 composite lightcurves spanning more than 12 years, from April 2002 to August 2014. In order to increase the reliability of our results we used 11 additional individual lightcurves obtained in October and November 2004 by Reddy et al. (2005) and by Licchelli (2006).
Table 1. Observational circumstances of (1459) Magnya's lightcurves.

\begin{tabular}{|c|c|c|c|c|c|}
\hline Date & $\begin{array}{l}\text { Start } \\
\text { UT }\end{array}$ & $\begin{array}{l}\text { End } \\
\text { UT }\end{array}$ & $\operatorname{Mag} V$ & $\begin{array}{l}\text { Solar } \\
\text { phase }\end{array}$ & Obs./tel. \\
\hline 2002-Apr.-22 & 0.37 & 4.21 & 15.7 & 1.6 & OPD/1.6 m \\
\hline 2002-Apr.-23 & 1.99 & 5.20 & 15.7 & 1.8 & $\mathrm{OPD} / 1.6 \mathrm{~m}$ \\
\hline 2004-Aug.-11 & 4.96 & 8.46 & 14.7 & 23.6 & $\mathrm{OPD} / 0.6 \mathrm{~m}$ \\
\hline 2004-Aug.-12 & 5.02 & 7.94 & 14.7 & 23.5 & $\mathrm{OPD} / 0.6 \mathrm{~m}$ \\
\hline 2004-Sep.-21 & 6.51 & 8.11 & 13.9 & 13.6 & OPD/0.6 m \\
\hline 2004-Sep.-22 & 4.45 & 8.17 & 13.9 & 13.3 & $\mathrm{OPD} / 0.6 \mathrm{~m}$ \\
\hline 2004-Oct.-17 & 19.35 & 21.55 & 13.3 & 3.4 & $\mathrm{FO} / 0.21 \mathrm{~m}^{a}$ \\
\hline 2004-Oct.-17 & 23.42 & 1.38 & 13.3 & 3.4 & $\mathrm{FO} / 0.21 \mathrm{~m}^{a}$ \\
\hline 2004-Oct.-20 & 19.45 & 20.32 & 13.3 & 3.3 & $\mathrm{FO} / 0.21 \mathrm{~m}^{a}$ \\
\hline 2004-Oct.-22 & 20.34 & 22.38 & 13.3 & 3.6 & $\mathrm{FO} / 0.21 \mathrm{~m}^{a}$ \\
\hline 2004-Oct.-22 & 23.25 & 1.30 & 13.3 & 3.6 & $\mathrm{FO} / 0.21 \mathrm{~m}^{a}$ \\
\hline 2004-Oct.-23 & 20.06 & 21.62 & 13.3 & 3.8 & $\mathrm{FO} / 0.21 \mathrm{~m}^{a}$ \\
\hline 2004-Oct.-30 & 13.98 & 15.56 & 13.5 & 6.2 & $\mathrm{BO} / 0.35 \mathrm{~m}^{b}$ \\
\hline 2004-Nov.-03 & 10.58 & 15.88 & 13.6 & 7.8 & $\mathrm{BO} / 0.35 \mathrm{~m}^{b}$ \\
\hline 2004-Nov.-06 & 2.48 & 9.27 & 13.7 & 8.9 & $\mathrm{BD} / 0.66 \mathrm{~m}^{c}$ \\
\hline 2004-Nov.-07 & 3.23 & 5.03 & 13.7 & 9.3 & $\mathrm{BD} / 0.66 \mathrm{~m}^{c}$ \\
\hline 2004-Nov.-09 & 12.71 & 16.18 & 13.8 & 10.3 & $\mathrm{BO} / 0.35 \mathrm{~m}^{b}$ \\
\hline 2008-May-06 & 23.33 & 7.16 & 15.4 & 1.4 & OPD/0.6 m \\
\hline 2008-May-08 & 5.44 & 6.25 & 15.4 & 1.1 & $\mathrm{OPD} / 0.6 \mathrm{~m}$ \\
\hline 2014-Мay-08 & 6.30 & 7.45 & 15.5 & 9.8 & OASI $/ 1.0 \mathrm{~m}$ \\
\hline 2014-May-23 & 6.01 & 7.34 & 15.2 & 6.1 & $\mathrm{OASI} / 1.0 \mathrm{~m}$ \\
\hline 2014-Jun.-04 & 0.81 & 5.71 & 15.1 & 4.2 & OASI $/ 1.0 \mathrm{~m}$ \\
\hline 2014-Jun.-30 & 21.24 & 3.03 & 15.3 & 9.6 & OASI $/ 1.0 \mathrm{~m}$ \\
\hline 2014-Jul.-20 & 21.55 & 2.65 & 15.6 & 14.5 & $\mathrm{OASI} / 1.0 \mathrm{~m}$ \\
\hline 2014-Jul.-28 & 21.18 & 23.18 & 15.7 & 16.0 & OASI $/ 1.0 \mathrm{~m}$ \\
\hline 2014-Jul.-31 & 21.24 & 2.03 & 15.7 & 16.4 & OASI $/ 1.0 \mathrm{~m}$ \\
\hline 2014-Aug.-29 & 21.06 & 0.28 & 16.0 & 19.0 & OASI $/ 1.0 \mathrm{~m}$ \\
\hline
\end{tabular}

Notes. (a) R.P. Feynman Observatory, Gagliano del Capo, Italy. (b) Barfold Observatory, Glenhope, Victoria, Australia. ${ }^{(c)}$ Badlands Observatory, Quinn, South Dakota, USA.

The observational circumstances for all the used lightcurves are given in Table 1 where for each date is given the starting and ending time (UT) of the observations, the asteroid visual magnitude, the solar phase, and the observatory/telescope.

\section{Pole and shape solution}

The pole direction and shape model for (1459) Magnya were obtained using the lightcurve inversion method by Kaasalainen and collaborators (Kaasalainen \& Torppa 2001; Kaasalainen et al. 2001). The method also allowed a better estimation of the asteroid rotational period. In the computations were used a total of 27 lightcurves. The magnitudes were considered as relative, even when absolute, for simplicity and because of the different filters used in the observation, in which case the use of relative magnitude helps the fit among the diverse lightcurves.

In addition to the lightcurves, the method requires other information such as the ecliptic astrocentric cartesian coordinates $X, Y, Z$ of the Sun and of the Earth, in $\mathrm{AU}$, and an initial estimation for the pole direction and rotational period. The lightscattering behavior is described as a combination of LommelSeeliger and Lambert models (Kaasalainen et al. 2001). The surface reflectance $S$ as a function of the angle of incidence $i$ and the angle of emergence $e$ is given by

$S\left(\mu, \mu_{0}, \alpha\right)=f(\alpha)\left[S_{\mathrm{LS}}\left(\mu, \mu_{0}\right)+c S_{\mathrm{L}}\left(\mu, \mu_{0}\right)\right]$, 

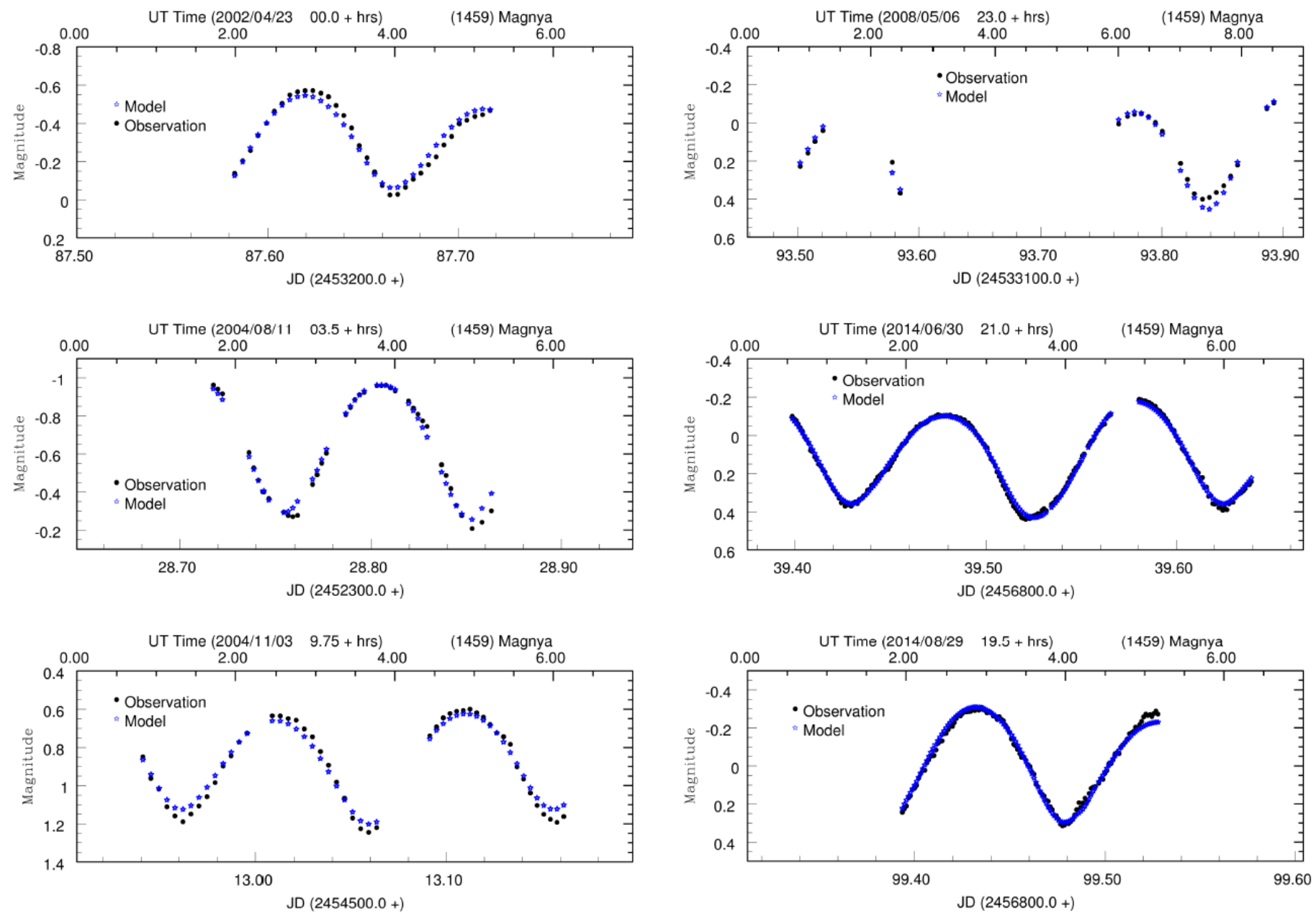

Fig. 1. Observed and modeled solution with pole direction $\lambda=159 \pm 10^{\circ}$ and $\beta=-75 \pm 10^{\circ}$. Black points and blue stars represent the observations and the model, respectively.

where $\mu_{0}=\cos i, \mu=\cos e$, and $S_{\mathrm{LS}}$ and $S_{\mathrm{L}}$ are the LommelSeeliger and the Lambert term, respectively, given by

$S_{\mathrm{LS}}\left(\mu, \mu_{0}\right)=\frac{\mu \mu_{0}}{\mu+\mu_{0}}$

and

$S_{\mathrm{L}}\left(\mu, \mu_{0}\right)=\mu \mu_{0}$,

and $c$ is the weight factor. No phase function $f(\alpha)$ is used in our case. The model is obtained from the best fit between the observed and modeled lightcurves by means of an iterative process. The equation that defines the fit is given by

$\chi_{\mathrm{rel}}^{2}=\sum_{1}\left\|\frac{L_{\mathrm{obs}}^{i}}{\bar{L}_{\mathrm{obs}}^{i}}-\frac{L^{i}}{\bar{L}^{i}}\right\|$,

where $L_{\mathrm{obs}}^{i}$ and $L^{i}$ are observed and modeled lightcurves, while $\bar{L}_{\text {obs }}^{i}$ and $\bar{L}^{i}$ are the average brightness values.

Applying the method to the 27 lightcurves of (1459) Magnya, a refined period of $4.679100 \pm 0.000005 \mathrm{~h}$ is obtained. Furthermore, independently of the given initial values, a rapid convergence is obtained for the pole direction defined by $\lambda=159 \pm 10^{\circ}$ and $\beta=-75 \pm 10^{\circ}$, ecliptic longitude and latitude, respectively. No convergence to the opposite pole direction, at $\lambda=340^{\circ}$ and $\beta=-75^{\circ}$, has been obtained. Figure 1 shows how the selected solution fits the lightcurves for different years and/or geometric configurations. In the plots the black points represent the observations and the blue stars the model.

The shape model for (1459) Magnya is represented by a convex polyhedron with triangular facets as shown in Fig. 2 in three different positive views in the asteroid's Cartesian frame $X, Y$, and $Z$.

\section{Discussion}

The obtained model for (1459) Magnya, Fig. 2, indicates a slightly elongated shape, responsible for the 0.6-0.7 amplitude of the lightcurves, with the rotational axis along the smallest axis. No surface structures are visible, as expected because of the almost symmetrical two-peaked lightcurves as shown in Almeida et al. (2004).

Regarding the composition of (1459) Magnya's surface, after the discovery of its basaltic nature (Lazzaro et al. 2000) several spectral observations have been performed by diverse authors all confirming the presence of the two absorption bands, at 0.92$0.94 \mu \mathrm{m}$ and at $1.98-2.0 \mu \mathrm{m}$, indicative of pyroxene. Because we know the direction of the pole, it is now possible to determine which regions of the asteroid were sampled by these diverse observations. In Table 2 are summarized the viewing geometry for each date of observation, i.e., the mean value of the solar phase, the $V$ magnitude, and the aspect angle. It is important to remember that the aspect angle is the angle between the 


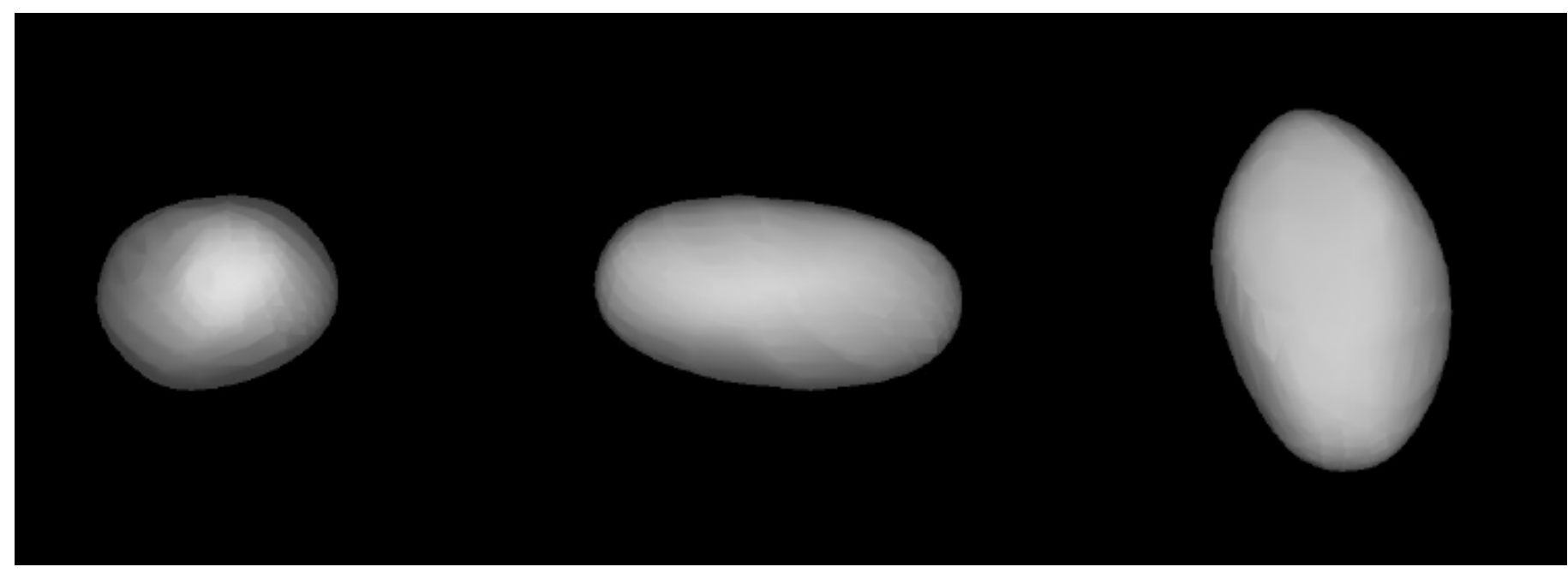

Fig. 2. (1459) Magnya model: $X, Y$, and $Z$ positive views in the asteroid's cartesian frame.

Table 2. Visible configurations at the time of the diverse spectroscopic observations.

\begin{tabular}{lcccc}
\hline \hline Date & $\begin{array}{c}\text { Solar } \\
\text { phase }\left(^{\circ}\right)\end{array}$ & $V_{\text {mag }}$ & $\begin{array}{c}\text { Aspect } \\
\text { angle }\left(^{\circ}\right)\end{array}$ & Reference \\
\hline Sep. 1998 & 13 & 14 & 50 & 1 \\
Nov.-Dec. 1999 & $16-9$ & 15 & 90 & 1 \\
Jan. 2000 & 10 & 15 & 85 & 2 \\
Feb. 2001 & 6 & 16 & 152 & 3 \\
Mar. 2002 & 8 & 16 & 155 & 4 \\
\hline
\end{tabular}

References. (1) Lazzaro et al. (2000); (2) Burbine \& Binzel (2002); (3) DeMeo et al. (2009); (4) Hardersen et al. (2004).

line of sight of the observer and the positive direction of the spin axis.

From the values given in the table we can see that the observations made between Sep. 1998 and Jan. 2000 (Lazzaro et al. 2000; Burbine \& Binzel 2002), probed the equatorial region of the asteroid because the aspect angle was about $90^{\circ}$. Although in the case of the ESO observations (Sep. 1998) two visible spectra were obtained, these were acquired within an interval of $15 \mathrm{~min}$, which makes it impossible to assess the presence of rotational variations.

The observations obtained in 2001 and 2002 (DeMeo et al. 2009; Hardersen et al. 2004), on the other hand, were obtained at an aspect angle of $150^{\circ}$, thus sampling the region around the south pole. In the case of the observations by Hardersen et al. (2004) in addition to the mineralogical analysis of the asteroid surface these were also intended to sample the asteroid surface during its rotation in order to search for possible compositional variations. Although at the time of their observations no period had been published, the observations were performed over a $4.5 \mathrm{~h}$ interval which guarantees that nearly $95 \%$ of the asteroid's visible surface was observed. However, it is now clear that the viewing geometry was such that the visible surface was nearly unchanged during the observations. In this sense, the minor variations detected in the spectra could be due to the nearly poleon configuration and not necessarily to a global homogeneous surface.

In Sep. 2004, we performed visible spectroscopic observations of (1459) Magnya at the OPD intending to search for spectral variations over several nights. Again, the viewing geometry was nearly pole-on, at an aspect angle of $20^{\circ}$, with the only difference that the north pole was sampled. Unfortunately, these spectra are in the visible $(0.55-0.92 \mu \mathrm{m})$, making any comparison with the observations done by Hardersen et al. (2004) difficult.

The information on the homogeneity (or non-homogeneity) of an asteroid surface does not provide conclusive evidence of its origin. However, in the case of (1459) Magnya we can derive important clues since its basaltic surface implies a differentiation process and, thus, an originally layered object. If we observe a non-homogeneous surface this could indicate either that we are seeing different layers of the original object, or that it is a fragment from a larger layered object, such as (4) Vesta. On the other hand, a surface homogeneous one could indicate that (1459) Magnya's surface is intact, corroborated also by its almost ellipsoidal shape. If this indeed is the case, we would be facing the interesting scenario of the differentiation of small objects.

To conclude, we would like to note that the results presented here indicate the need to schedule new visible and near-infrared observation of (1459) Magnya in order to correctly assess the degree of homogeneity of its surface. It is noteworthy that at the next opposition, in October 2015, the viewing geometry of (1459) Magnya will be optimal, with the aspect angle at about $95^{\circ}$, allowing the sampling of the equatorial region. An opportunity that cannot be missed!

Acknowledgements. The authors acknowledge CNPq for the fellowships and FAPERJ for the support to D.L., through project number E-26-102.967/2011. We thank V. Reddy and D. Licchelli for making their data available to us. Finally, we are gratefully to the IMPACTON team, in particular, T. Rodrigues, J. M. Carvano, F. Roig and R. de Souza, for all the efforts to make the OASI operative.

\section{References}

Almeida, R., Angeli, C. A., Duffard, R., \& Lazzaro, D. 2004, A\&A, 415, 403

Alvarez-Candal, A., Duffard, R., Lazzaro, D., \& Michtchenko, T. 2006, A\&A, 459, 969

Binzel, R. P., \& Xu, S. 1993, Science, 260, 186

Binzel, R. P., Masi, G., \& Foglia, S. 2006, in BAAS 38, AAS/Division for Planetary Sciences Meeting Abstracts, 38, 627

Burbine, T. H., \& Binzel, R. P. 2002, Icarus, 159, 468

Burbine, T. H., Buchanan, P. C., Binzel, R. P., et al. 2001, Meteor. Planet. Sci., 36,761 


\section{J. S. Silva and D. Lazzaro: Pole and Shape of (1459) Magnya $(R N)$}

Cruikshank, D. P., Tholen, D. J., Bell, J. F., Hartmann, W. K., \& Brown, R. H. 1991, Icarus, 89, 1

Delbo, M., Gai, M., Lattanzi, M. G., et al. 2006, Icarus, 181, 618

DeMeo, F. E., Binzel, R. P., Slivan, S. M., \& Bus, S. J. 2009, Icarus, 202, 160

Drake, M. J. 2001, Meteor. Planet. Sci., 36, 501

Florczak, M., Lazzaro, D., \& Duffard, R. 2002, Icarus, 159, 178

Hammergren, M., Gyuk, G., \& Puckett, A. W. 2007, in Am. Astron. Soc. Meet. Abst. 210, BAAS, 39, 203

Hardersen, P. S., Gaffey, M. J., \& Abell, P. A. 2004, Icarus, 167, 170

Kaasalainen, M., \& Torppa, J. 2001, Icarus, 153, 24

Kaasalainen, M., Torppa, J., \& Muinonen, K. 2001, Icarus, 153, 37

Lazzaro, D., Michtchenko, T., Carvano, J. M., et al. 2000, Science, 288, 2033

Lazzaro, D., Angeli, C. A., Carvano, J. M., et al. 2004, Icarus, 172, 179

Licchelli, D. 2006, Minor Planet Bull., 33, 11
McCord, T. B., Adams, J. B., \& Johnson, T. V. 1970, Science, 168, 1445

Michtchenko, T. A., Lazzaro, D., Ferraz-Mello, S., \& Roig, F. 2002, Icarus, 158, 343

Moskovitz, N. A., Lawrence, S., Jedicke, R., et al. 2008, ApJ, 682, L57

Moskovitz, N. A., Willman, M., Burbine, T. H., Binzel, R. P., \& Bus, S. J. 2010, Icarus, 208, 773

Reddy, V., Dyvig, R. R., Heathcote, B. D., \& Pravec, P. 2005, in 36th Annual Lunar and Planetary Science Conf., eds. S. Mackwell, \& E. Stansbery, 36, 1394

Roig, F., \& Gil-Hutton, R. 2006, Icarus, 183, 411

Russell, C. T., Raymond, C. A., Coradini, A., et al. 2012, Science, 336, 684

Ruzicka, A., Snyder, G. A., \& Taylor, L. A. 1997, Meteor. Planet. Sci., 32, 825

Xu, S., Binzel, R. P., Burbine, T. H., \& Bus, S. J. 1995, Icarus, 115, 1 